\title{
Management of primary rhegmatogenous retinal detachment with inferior breaks
}

\author{
A Sharma, V Grigoropoulos, T H Williamson
}

Br J Ophthalmol 2004;88:1372-1375. doi: 10.1136/bjo.2003.041350

Background: Rhegmatogenous retinal detachments (RRD) with inferior breaks are usually treated by scleral buckling (SB) or pars plana vitrectomy (PPV) or a combination of both methods. However, applying a SB during PPV may produce a risk of choroidal haemorrhage. Following a recent pilot study showing that such cases can be safely treated by PPV without $S B$ the authors re-examined their management of RRD in which inferior breaks were present.

Methods: All patients had a detached vitreous and a complex configuration of retinal breaks. A case-control study was performed to analyse the surgical methods and results of PPV on 48 consecutive patients with RRD associated with inferior breaks and 48 age/sex matched controls who underwent PPV for RRD without inferior breaks. Exclusion criteria were giant retinal tears, retinal dialysis, trauma, proliferative vitreoretinopathy (PVR) grade B or higher, schisis detachments, and eyes that had been operated previously for RRD. A simple algorithm was followed to manage patients with inferior breaks. All patients underwent a standard three port PPV with intraocular gas tamponade without supplementary SB. Patients were asked to posture face up or right or left side down for 1 week.

Results: 39 of the 48 patients (81.3\%) with inferior breaks were treated successfully with one operation. 41 of 48 patients $(85.4 \%)$ control patients achieved primary success. The final success rate was $95.8 \%$ in both groups. There was no statistical difference between the two groups. When all the cases of RRD were analysed (including external plomb/nondrain procedures) the primary success rate was $89 \%$ and final success rate $97.5 \%$.

Conclusions: This study has shown that acceptable success rates can be achieved using PPV alone to treat RRD with inferior breaks. Complications are minimised and patients in this high risk group have an $81 \%$ chance of primary success. Pars plana vitrectomy and gas will successfully reattach the retina and a supplementary SB, to support the inferior retina, is unnecessary as the intraocular gas, and face up or, right or left side down positioning will tamponade breaks satisfactorily.

\section{A} lthough rhegmatogenous retinal detachment (RRD) surgery achieves success rates of $80 \%$ or over, improvement in success rates has been minimal in the past 20 years. ${ }^{1}$ Further development of surgical methods is required. Currently a multitude of techniques are employed with no overall consensus on the most effective methodology. ${ }^{1-5}$ Scleral buckling $(\mathrm{SB})^{67}$ and pars plana vitrectomy $(\mathrm{PPV})^{8-12}$ are most commonly used either alone $^{13}$ or in combination. ${ }^{9}$ The selection of the surgical approach depends on many factors, one of which is the position of the breaks in the retina. The majority of RRDs are associated with superior or midline breaks, ${ }^{14}$ which can be treated with either conventional SB alone or PPV alone. However, inferior breaks present a surgical challenge so that surgeons will often combine a PPV with SB. The SB is used to produce an inferior indent, ${ }^{9}$ because it is believed that intraocular tamponade with gas cannot provide direct support to the inferior retina. The practice of SB during PPV is not free of risks, particularly because hypotony of the vitrectomised eye during placement of the buckle may increase the risk of choroidal haemorrhage. ${ }^{15}$ It is uncertain whether the SB during PPV is necessary. The results of Campo et al have suggested that adequate retinal reattachment rates can be obtained by using PPV and gas only, albeit with extensive peripheral $360^{\circ}$ laser therapy. ${ }^{16}$ The authors of that study do not mention the position of breaks but must surely have had some patients with inferiorly placed breaks. If PPV can be used alone it may be possible to avoid other complications associated with SBfor example, scleral perforation, refractive changes, ${ }^{17}$ diplopia, ${ }^{18} 19$ explant erosion or infection, ${ }^{18}{ }^{20}$ decreased retinal blood flow, and anterior segment ischaemia. ${ }^{21} 22$

In a small pilot study we determined that buckling of inferior breaks during vitrectomy was unnecessary and that a success rate of $89 \%$ could be achieved. ${ }^{23}$ This encouraged a change in practice. However, analysis of a larger sample size was required to ensure that this success rate could be maintained. In this study we investigated the results of all patients with RRD with inferior breaks, operated upon by $\mathrm{PPV}$, in a 36 month period in our service, to assess the surgical approach and retinal reattachment rate.

\section{METHODS}

We identified those patients with retinal breaks between the 4 and $80^{\prime}$ clock meridians and defined these patients as having inferior breaks. We collected data on 48 patients who had undergone PPV for RRD with inferior breaks over a period of 3 years. Information on the retinal detachment, number, and location of retinal breaks was collected from a vitreoretinal database that is continuously updated with the patient's details (retina surgery freeware using Microsoft Access 97, specifically designed by one of the authors, THW). Using these data and reviewing clinical notes, where validation of results was required, all patients that were operated by PPV for RRD with inferior retinal breaks between January 2001 and December 2003 were studied. All patients had slit lamp examination including biomicroscopy of the vitreous and retina, and indirect ophthalmoscopy. The existence of posterior vitreous detachment was diagnosed on the basis of the presence of a Weiss ring, by ultrasound examination, or by the presence of $U$ tears. Only data from the first presenting eye of any patient were used.

Abbreviations: PPV, pars plana vitrectomy; PVR, proliferative vitreoretinopathy; RRD, rhegmatogenous retinal detachments; SB, scleral buckling; SRF, subretinal fluid 


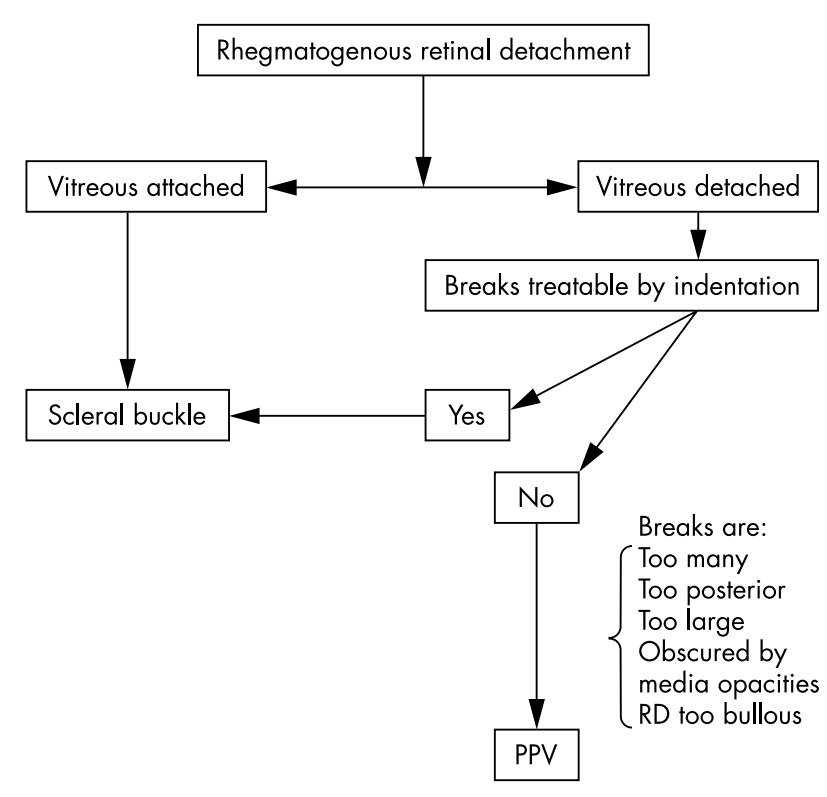

Figure 1 Treatment protocol for patients with inferior breaks (PPV, pars plana vitrectomy).

We followed an algorithm to plan surgery (fig 1) and 48 patients with inferior breaks met our criteria to undergo vitrectomy, and were included in the study. It has been shown that better visual results are obtained in the under 60 year age group after RRD surgery. ${ }^{14}$ Slightly more men than women present with RRD and we therefore selected 48 age and sex matched patients, who had RRD with breaks outside the inferior 4-8 o'clock area, from the database to act as controls. Exclusion criteria were giant retinal tears, retinal dialysis, trauma, proliferative vitreoretinopathy (PVR) grade $B$ or higher, schisis detachments, and eyes that had been operated previously for RRD. All patients underwent a standard three port pars plana vitrectomy, using the Oculus BIOM viewing system (Wetzler, Germany), with deep scleral indentation to identify all breaks. All patients had either trans-scleral cryopexy or endolaser with internal drainage of subretinal fluid (SRF) and internal gas tamponade using
$\mathrm{C}_{3} \mathrm{~F}_{8}$ or $\mathrm{SF}_{6}$. Four patients and five controls ultimately required silicone oil tamponade. No scleral buckling was used in either group and all patients were given written instructions, with clear diagrams, on how to posture. This was face up or right or left cheek down, depending on the position of the breaks, for 50 minutes in the hour for 7 days postoperatively, without the use of any posturing aids.

Postoperatively all patients had their best corrected visual acuities recorded as well as the anatomical result of surgery, status of the macula, and any complications. Statistical analysis was performed using a two tailed Student's $t$ test or Fisher's exact test.

\section{RESULTS}

Examples of RRD with inferior breaks are shown in figure 2. Patient details are shown in table 1. Success rates are shown in table 2 and figure 3. The mean age ( 58 years) and sex distribution (36 males, 12 females) was equal in both patient groups. Mean follow up was 6 months (range 6 weeks36 months). Control patients had a mean of 2.0 quadrants of detached retina and the inferior breaks group 2.3 (statistically no difference using Fisher's exact test). There were 29 macula off and 19 macula-on patients in the control group. In the inferior breaks group 22 were macula-on and 25 maculaoff and in one patient the macula status was not recorded. The mean number of breaks per eye in the controls was 2.6 and in the inferior breaks patients 4.6 , which was statistically significant $(\mathrm{p}<0.001)$.

The majority of the patients improved postoperatively as would be expected. In the inferior breaks group (fig 4) vision improved from a mean preoperative logMAR acuity of 0.8 $(6 / 38)$ to $0.54(6 / 20)$. For the control group vision improved from a mean logMAR of $1.04(6 / 60)$ to 0.52 (6/20) following surgery. No patient in either group suffered from any sight threatening complications such as choroidal haemorrhage and none developed pupil block glaucoma. No formal assessment of cataract progression was made but, out of 21 controls with clear lenses preoperatively, three were documented to have cataract during their postoperative follow up. For the inferior breaks group nine developed cataract out of 25 who had clear lenses. This did not reach statistical significance using Fisher's exact test and we were not aware of any difference in cataract surgery postoperatively between
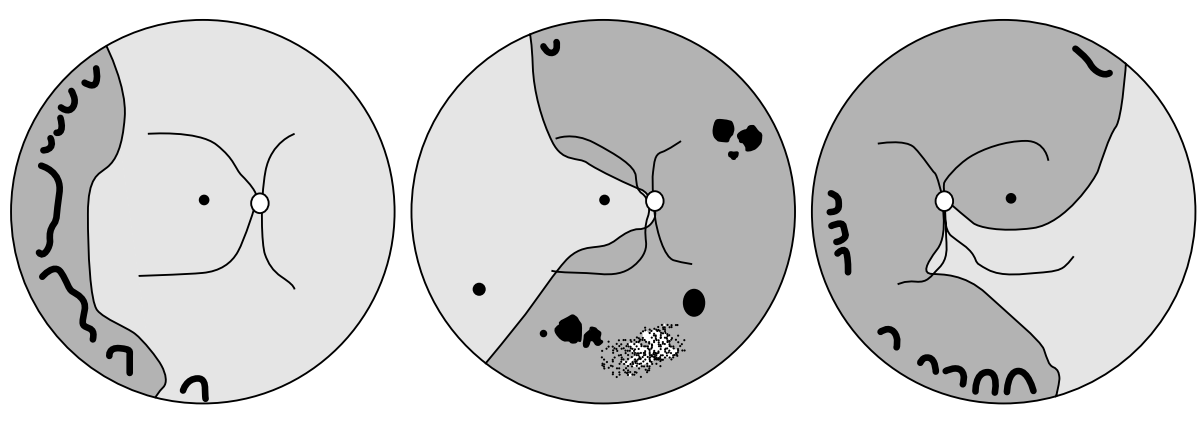

Figure 2 Retinal drawings showing examples of retinal detachments with inferior breaks treated by PPV.

Detached retina in dark grey, attached retina in light grey, breaks in black, and lattice are shown.

Table 1 Patient details

\begin{tabular}{|c|c|c|c|c|c|c|}
\hline & $\begin{array}{l}\text { Mean age } \\
\text { (years) }\end{array}$ & $M: F$ & $\begin{array}{l}\text { Mean no of } \\
\text { quadrants RD }\end{array}$ & $\begin{array}{l}\text { Mean no of } \\
\text { breaks per eye (SD) }\end{array}$ & Pseudophakia & Aphakia \\
\hline $\begin{array}{l}\text { Controls without inferior } \\
\text { breaks }\end{array}$ & 58.1 & $36: 12$ & 2.0 & $2.6(2.2)$ & 9 & 2 \\
\hline Patients with inferior breaks & 58.1 & $36: 12$ & 2.3 & $4.6(3.2) p<0.001$ & 12 & 0 \\
\hline
\end{tabular}


Table 2 Success rates

\begin{tabular}{llll}
\hline & $\begin{array}{l}\text { Superior breaks } \\
\text { group (controls) }\end{array}$ & $\begin{array}{l}\text { Inferior breaks group } \\
\text { (patients) }\end{array}$ & $\begin{array}{l}\text { Overall data from 279 } \\
\text { RRD cases }\end{array}$ \\
\hline $\begin{array}{l}\text { Primary success rate } \\
\text { Final success rate }\end{array}$ & $41 / 48(85.4 \%)$ & $39 / 48(81.3 \%)$ & $248 / 279(88.9 \%)$ \\
\hline
\end{tabular}

Primary success rates are similar in the superior and inferior breaks groups $(p=0.63)$.

The third column shows our overall performance for all primary RRDs at our unit (excluding PVR, giant tears,

dialyses, and retinoschisis). The patients with inferior breaks represent a higher risk group when compared to our

overall data.

the two groups. We would expect to see more cataract development with a longer duration of follow up.

Primary success rates in the inferior break group was $81.3 \%$ $(39 / 48)$ and in the control group $85.4 \%$ (41/48). This difference did not reach statistical significance $(p=0.63)$. Final success rates were $95.8 \%$ in both groups (table 2 ). When our algorithm was used for 279 patients presenting to our unit with primary RRD, our primary success rate was $88.9 \%$ and final success rate $97.5 \%$. The poorer results from the study group with inferior breaks and their controls reflects that more simple retinal detachment cases were treated by a non-drain procedure.

\section{DISCUSSION}

The past three decades has seen the greater use of pars plana vitrectomy over scleral buckling for uncomplicated RRD cases. ${ }^{1}$ It is often combined with scleral buckling but the precise role of scleral buckling during vitrectomy procedures has yet to be clarified. To minimise the attendant risks of buckling on vitrectomised eyes, we explored the effect of eliminating scleral buckling for inferiorly located breaks during vitrectomy for RRD. We have shown that adequate success rates can be achieved when repairing RRD, with inferior breaks, using vitrectomy without scleral buckling. Our primary success rate was $81.3 \%$ for the inferior break group and $85.4 \%$ for the control group without inferior breaks. Final success rates were identical for both groups $(95.8 \%)$. The difference in primary success figures did not reach statistical significance. These figures are comparable with those obtained by other workers ${ }^{913}{ }^{14}$ but it has to be stressed that patient groups and surgical techniques are not directly comparable. When compared with success rates nationwide ${ }^{25}$ our figures compare well with those of vitreoretinal specialists operating on unselected cases (82\% primary success and $91.5 \%$ success at 3 months). Specialists operating on RRD with horseshoe tears achieved a primary reattachment rate of $80 \%$ in this national audit.

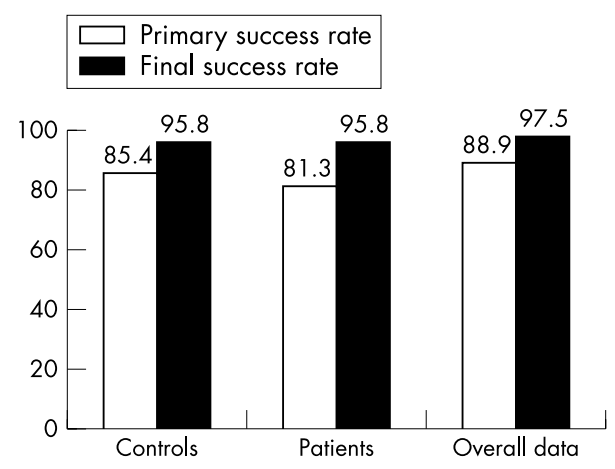

Figure 3 Success rates (\% cases). Success rates are similar for the patients with inferior breaks and controls $(p=0.63)$. Our overall data are calculated from 279 primary RRDs undergoing PPV or SB, excluding cases with PVR, giant tears, dialyses, and retinoschisis.
We have previously shown in a pilot study of uncomplicated $\mathrm{RRD}^{23}$ that scleral buckling for inferior retinal breaks is not necessary for success. Our present study confirms this finding in a larger group. It may be argued that our primary success rates could be better, but this study excluded the more straightforward types of RRD, without inferior breaks, that could be repaired using a conventional external buckle/ non-drain procedure. When these simpler types of RRD are included our primary success rate has been calculated as $89 \%$ and final success rate $97.5 \%$, which compare well with other centres offering a specialist vitreoretinal service (80-90\% primary success and $97 \%$ final success ). ${ }^{526} 27$ In one study the main preoperative risk factor associated with failure of primary reattachment surgery was breaks not closeable by indentation. ${ }^{5}$ This supports our algorithm of using PPV for such cases. Studies on selected ${ }^{24}$ and unselected ${ }^{13}$ RRD repair using vitrectomy reveal primary success rates of $79 \%$ that drop to $64 \%$ if patients have preoperative PVR. However final success rates reach $92-93 \%$.

It can be seen from table 1 that patients with inferior breaks had more breaks per eye. Naturally some patients with inferior breaks would also have had superior ones. Having breaks both superiorly and inferiorly meant that the inferior break patients would, on average, have more breaks per eye (4.6) compared with the controls (2.6) and this was statistically significant. This did not however influence our management as all patients underwent the same technique. It may be that RRD with inferior breaks performs less well because it is more prone to the effects of PVR, which is more likely to occur inferiorly.

Over the years PPV has been used without scleral buckling for ever more complicated RRDs such as aphakia and pseudophakia ${ }^{17}{ }^{24}$ with satisfactory results. Inevitably some patients with inferior retinal breaks would have been included in these studies but rarely have they received specific attention as a separate group. A recent study ${ }^{28}$ used pneumatic retinopexy with inverted head posturing to treat patients with inferior retinal breaks. Previously, pneumatic retinopexy was thought to be contraindicated in RRDs with

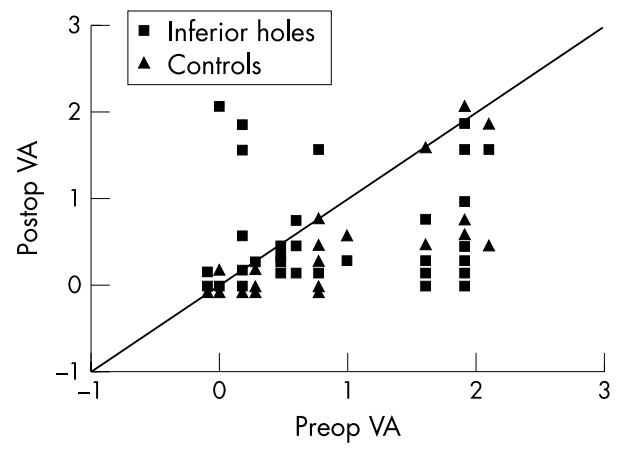

Figure 4 Change in vision (logMAR VA). All points below the line of equality show an improvement in vision. No difference was observed between the inferior breaks group and controls. 
inferior breaks ${ }^{29}{ }^{30}$ but, as Chang's study showed, inverted posturing achieved primary success in eight of 11 subjects $(82 \%)$ and final success in all subjects. The posturing involved is quite extreme and many patients would not be suitable. We can conclude, however, that inferior breaks can be managed as superior ones provided tamponade is maintained for long enough for retinopexy to take effect.

While the success rates in both groups marginally favoured those who had no inferior breaks (85.4\% $v$ 81.3\%) the difference was not statistically significant. In conclusion, we have demonstrated that a "non-buckle" procedure for RRDs with inferior breaks can produce an $81.3 \%$ primary success and a $95.8 \%$ final success rate, while perhaps removing the extra risks of applying a buckle to a vitrectomised eye during PPV. Our algorithm reserves PPV for more complex cases and provides good success rates for retinal detachments while minimising risk.

\section{Authors' affiliations}

A Sharma, V Grigoropoulos, T H Williamson, Department of Ophthalmology, St Thomas's Hospital, Lambeth Palace Road, London SE1 7EH, UK

Correspondence to: Tom H Williamson, Department of Ophthalmology, St Thomas's Hospital, Lambeth Palace Road, London SE1 7EH, UK; tom@retinasurgery.co.uk

Accepted for publication 30 March 2004

\section{REFERENCES}

1 Minihan M, Tanner V, Williamson TH. Primary rhegmatogenous retinal detachment: 20 years of change. Br J Ophthalmol 2001;85:546-8.

2 Chignell AH, Fison LG, Davies EW, et al. Failure in retinal detachment surgery. Br J Ophthalmol 1973;57:525-30.

3 Sharma T, Challa JK, Ravishankar KV, et al. Scleral buckling for retinal detachment. Predictors for anatomic failure. Retina 1994;14:338-43

4 Wilkinson CP, Bradford RH Jr. Complications of draining subretinal fluid. Retina 1984;4:1-4.

5 Sullivan PM, Luff AJ, Aylward GW. Results of primary retinal reattachment surgery: a prospective audit. Eye 1997;11(Pt 6):869-71.

6 Lincoff H, McLean JM. Modifications to the Custodis procedure. II. A new silicone implant for large tears. Am J Ophthalmol 1967;64:877-9.

7 Rubin ML, Fitzgerald CR. The episcleral partial-thickness sponge for scleral buckling. Mod Probl Ophthalmol 1974; 12:495-501.

8 Ah-Fat FG, Sharma MC, Majid MA, et al. Trends in vitreoretinal surgery at a tertiary referral centre: 1987 to 1996. Br J Ophthalmol 1999;83:396-8.
9 Gartry DS, Chignell AH, Franks WA, et al. Pars plana vitrectomy for the treatment of rhegmatogenous retinal detachment uncomplicated by advanced proliferative vitreoretinopathy. Br J Ophthalmol 1993;77:199-203.

10 Hakin KN, Lavin MJ, Leaver PK. Primary vitrectomy for rhegmatogenous retinal detachment. Graefes Arch Clin Exp Ophthalmol 1993;231:344-6.

11 Leaver $\mathbf{P}$. Expanding the role of vitrectomy in retinal reattachment surgery. Br J Ophthalmol 1993;77:197.

12 Wong D, Billington BM, Chignell $\mathrm{AH}$. Pars plana vitrectomy for retinal detachment with unseen retinal holes. Graefes Arch Clin Exp Ophthalmol 1987;225:269-71.

13 Heimann H, Bornfeld N, Friedrichs W, et al. Primary vitrectomy without scleral buckling for rhegmatogenous retinal detachment. Graefes Arch Clin Exp Ophthalmol 1996;234:561-8.

14 Tani P, Robertson DM, Langworthy A. Prognosis for central vision and anatomic reattachment in rhegmatogenous retinal detachment with macula detached. Am J Ophthalmol 1981;92:611-20.

15 Tabandeh H, Sullivan PM, Smahliuk P, et al. Suprachoroidal hemorrhage during pars plana vitrectomy. Risk factors and outcomes. Ophthalmology 1999; 106:236-42.

16 Campo RV, Sipperly JO, Sneed SR, et al. Pars plana vitrectomy without scleral buckle for pseudophakic retinal detachments. Ophthalmology 1999; 106:1811-5.

17 Goel R, Crewdson J, Chignell AH. Astigmatism following retinal detachment surgery. Br J Ophthalmol 1983;67:327-9.

18 Arruga A. Motility disturbances induced by operations for retinal detachment. Mod Probl Ophthalmol 1977;18:408-14.

19 Fison PN, Chignell AH. Diplopia after retinal detachment surgery. Br J Ophthalmol 1987;71:521-5.

20 Flindall RJ, Norton EW, Curtin VT, et al. Reduction of extrusion and infection following episcleral silicone implants and cryopexy in retinal detachment surgery. Am J Ophthalmol 1971;71:835-7.

21 Kwartz J, Charles S, McCormack P, et al. Anterior segment ischaemia following segmental scleral buckling. Br J Ophthalmol 1994;78:409-10.

22 Ryan SJ, Goldberg MF. Anterior segment ischemia following scleral buckling in sickle cell hemoglobinopathy. Am J Ophthalmol 1971;72:35-50.

23 Tanner V, Miniham M, Williamson TH. Management of inferior breaks during pars plana vitrectomy for retinal detachement. $\mathrm{Br} J$ Ophthalmol 2001:85:480-2.

24 Escoffery RF, Olk RJ, Grand MG, et al. Vitrectomy without scleral buckling for primary rhegmatogenous retinal detachment. Am J Ophthalmol 1985;99:275-81.

25 Thompson JA, Snead MP, Billington BM, et al. National audit of the outcome of primary surgery for rhegmatogenous retinal detachment. II.Clinical outcomes. Eye 2002;16:771-7.

26 Johnson Z, Ramsay A, Cottrell D, et al. Triple cycle audit of primary retinal detachment surgery. Eye 2002;16:513-18.

27 Comer MB, Newman DK, George ND, et al. Who should manage primary retinal detachments? Eye 2000; 14:572-8.

28 Chang TS, Pelzek CD, Nguyen RL, et al. Inverted pneumatic retinopexy a method of treating retinal detachments associated with inferior retinal breaks. Ophthalmology 2003;110:589-94.

29 Hilton GF, Grizzard WS. Pneumatic retinopexy. A two-step outpatient operation without conjunctival incision. Ophthalmology 1986;93:626-41.

30 Tornambe PE, Hilton GF, Kelly NF, et al. Expanded indications for pneumatic retinopexy. Ophthalmology 1988;95:597-600. 